CERN-TH/99-138

DOE/ER/40561-55-INT99

$\mathrm{SCIPP} / 99 / 21$

\title{
The Long and Short of Nuclear Effective Field Theory Expansions
}

\author{
David B. Kaplan * \\ Institute for Nuclear Theory 351550, University of Washington, Seattle, WA 98195-1550, USA \\ dbkaplan@phys. washington.edu \\ James V. Steele \\ Department of Physics, The Ohio State University, Columbus, OH 43210-1106, USA \\ jsteele@mps.ohio-state.edu
}

\begin{abstract}
Nonperturbative effective field theory calculations for $N N$ scattering seem to break down at rather low momenta. By examining several toy models, we clarify how effective field theory expansions can in general be used to properly separate long- and short-range effects. We find that one-pion exchange has a large effect on the scattering phase shift near poles in the amplitude, but otherwise can be treated perturbatively. Analysis of a toy model that reproduces ${ }^{1} S_{0} N N$ scattering data rather well suggests that failures of effective field theories for momenta above the pion mass can be due to short-range physics rather than the treatment of pion exchange. We discuss the implications this has for extending the applicability of effective field theories.
\end{abstract}

May 14, 1999

*Address until June 30, 1999: Division TH, CERN, CH1211 Geneva 23, Switzerland. 


\section{INTRODUCTION}

Effective field theories (EFTs) allow for a description of low-energy data to arbitrary accuracy without detailed knowledge of the short-range interactions. The technique is most successful when there is a clear hierarchy of scales, for then the short-range physics only affects low-energy processes through weak irrelevant operators suppressed by powers of the short-distance scale. A good example is the $S U(3) \times S U(2) \times U(1)$ gauge theory for elementary particles, which is thought to be the effective low-energy formulation of a more complete theory. Experimental lower bounds on the momentum scale characterizing particular irrelevant operators from physics beyond the standard model are as high as $10^{16} \mathrm{GeV}$. In contrast, protein folding, for example, probably cannot benefit from an EFT analysis due to the absence of a large gap in the energy spectrum. Chiral perturbation theory is a successful EFT because the pion mass is small compared to other hadrons, as guaranteed in the chiral symmetry limit of QCD. Having an approximate symmetry to produce a small expansion parameter is important, because symmetry arguments can be used to unambiguously determine the importance of any particular process by counting powers of the small parameter.

Recently, there has been much interest in applying chiral perturbation theory to nuclear physics [1]. For low enough momentum, only interactions between pions and nucleons are relevant, with the effects of heavier hadrons (such as vector mesons and delta baryons) accounted for by higher dimension contact interactions. The primary goal is to uncover a systematic expansion of nuclear forces at low energy in powers of the pion mass and external momenta divided by a typical QCD scale $\Lambda_{S}$. This scale $\Lambda_{S}$ should be somewhat larger than $m_{\pi}$ for the expansion to be useful, but is unknown a priori.

A complication in nucleon dynamics that does not occur for chiral perturbation theory with zero or one nucleon is the appearance of a new long length scale, the nucleon-nucleon scattering length. The large value of the scattering length apparently arises from a fortuitous fine tuning of the short-distance physics. The situation is analogous to a condensed matter system near a phase transition, where interactions take on critical strengths, resulting in correlation lengths much longer than the lattice spacing. Similarly, the EFT for nucleons appears to be near a nontrivial critical point, and therefore the scaling dimensions of operators can be quite different from their naive dimension. This must indeed be the case, as all $N N$ interactions are irrelevant by naive dimensional analysis, yet they are quite strong in reality.

Weinberg's seminal papers [2] and their subsequent application [3 5] outline a way to apply EFT methods to nuclear physics. In the spirit of the Hamiltonian theory of Wilson with additional constraints imposed by chiral symmetry (in particular, the fact that the pion is derivatively coupled), the kernel of the Lippmann-Schwinger equation (i.e. the effective potential) is expanded in powers of $p / \Lambda_{S}$ and $m_{\pi} / \Lambda_{S}$ and then iterated to all orders. The scale $\Lambda_{S}$ is assumed not to vanish in the chiral limit and can be determined from fitting to data. In this approach, the way a given operator depends on $\Lambda_{S}$ is determined simply by dimensional analysis. In particular, the leading effects in this approach are due to one pion exchange (OPE) and a four nucleon contact interaction $\left(N^{\dagger} N\right)^{2}$, which both scale as $1 / \Lambda_{S}^{2}$.

However, since the interactions are tuned to a critical point, true and naive dimensions of operators differ and it is far from obvious that Weinberg's power counting is correct [6,7]. 
An alternative approach was therefore proposed by Kaplan, Savage, and Wise (KSW) [8], involving an expansion about the critical point corresponding to an infinite $s$-wave scattering length. In this analysis, one finds, for example, that only the $\left(N^{\dagger} N\right)^{2}$ operator is marginal, while OPE and other interactions are irrelevant and subleading. The effects of the leading contact interaction can be summed analytically, and the amplitude is expanded perturbatively in the higher dimension operators.

As we discuss below, the difference between the Weinberg and KSW expansions for $N N$ scattering at any given order only differ by terms considered higher order in the KSW approach, provided the renormalization scale in the former method is chosen to be sufficiently high. It is not clear that this correspondence will be true for systems with more than two nucleons. In any case, the KSW approach allows for a potentially great simplification compared to Weinberg's proposal, in particular for the case of many nucleon systems where iterating extended interactions to all orders is numerically intensive.

There has been much discussion and some controversy in the literature about which expansion scheme to use for an effective field theory of nuclear forces, and whether either one works at all [9]. Both schemes have had some success in the two- and three-nucleon sector [1], but it is not clear that they constitute an improvement over effective range theory [10], or the related (but more sophisticated) pionless nuclear EFT [11].

We address here the nature and implementation of the KSW expansion in the context of simple quantum mechanical models of the $N N$ interaction. We begin by analyzing a system with only short-range interactions and contrast the Weinberg and KSW expansions. We then consider arbitrary short-range interactions in conjunction with a caricature of single pion exchange, modeled by a delta-shell potential with radius $1 / m_{\pi}$. This model serves to illustrate how the effective field theory works when both long and short length scales are present. Based on our analytical treatment of this toy model, we develop a general method for computing the low-energy constants of the effective field theory. We then extend our analysis to more realistic examples and conclude with a discussion of the status of the KSW expansion.

\section{SHORT-RANGE INTERACTIONS}

We assume that interactions between two particles we will call "nucleons" can be described exactly by a potential $V=V_{S}+V_{L}$ consisting of a short- and long-range part. We simplify things even further in this section by taking the long-range potential $V_{L}$ to be zero. Within the context of field theory, it is most natural to express the scattering problem in terms of the Feynman scattering amplitude. Focusing exclusively on the ${ }^{1} S_{0}$ partial wave below, the amplitude is given by

$$
\mathcal{A} \equiv \frac{4 \pi}{M}\left(\frac{e^{2 i \widetilde{\delta}}-1}{2 i p}\right)=\frac{4 \pi / M}{p \cot \widetilde{\delta}-i p},
$$

where $p=\sqrt{M E_{c m}}$ is the center of mass momentum of the two nucleons and $\widetilde{\delta}$ is the phase shift for the short-range potential $V_{S}$ alone.

A successful EFT should describe the scattering amplitude in terms of an expansion in powers of momentum over the characteristic short-distance scale $\Lambda_{S}$. It was already evident 
fifty years ago that such an expansion is possible with the discovery of the effective range expansion [10], which expresses the quantity $p \cot \widetilde{\delta}$ as

$$
p \cot \widetilde{\delta}=-\frac{1}{\widetilde{a}}+\frac{1}{2} \widetilde{r}_{0} p^{2}+\sum_{n=2}^{\infty} \widetilde{v}_{n} p^{2 n},
$$

where $\widetilde{a}$ is the scattering length, $\widetilde{r}_{0}$ is the effective range, and the $\widetilde{v}_{n}$ are higher terms in the Taylor expansion. All the effective range parameters are expected to scale with the appropriate power of $\Lambda_{S}$ with the exception of $\widetilde{a}$, which can take on any value. We will focus on the case where the scattering length is abnormally large $|\widetilde{a}| \gg 1 / \Lambda_{S}$ (as occurs in actual $N N$ scattering), which signals the presence of a state that is weakly bound or almost bound.

\section{A. The Exact Solution of the Effective Theory}

The effective field theory description for this system is valid for momenta $p \lesssim \Lambda_{S}$ and consists solely of contact interactions in a derivative expansion between the nucleons. The fundamental $N N$ vertex at tree-level is

$$
\mathcal{A}_{\text {tree }}^{\mathrm{EFT}}=-\frac{4 \pi}{M} \widehat{C}\left(p^{2}\right)=-\frac{4 \pi}{M}\left(\widehat{C}_{0}+\widehat{C}_{2} p^{2}+\widehat{C}_{4} p^{4}+\ldots\right) .
$$

By using dimensional regularization and the PDS subtraction scheme, it is possible to compute the exact scattering amplitude for the EFT in terms of $\widehat{C}$, resulting in 8

$$
\mathcal{A}^{\mathrm{EFT}}=-\frac{4 \pi / M}{\widehat{C}^{-1}\left(p^{2} ; \mu\right)+\mu+i p},
$$

where $\mu$ is the renormalization scale. Comparing with Eq. (11) we see that the EFT formally agrees with the exact result, provided that the $\widehat{C}$ couplings are chosen to satisfy

$$
\frac{1}{\widehat{C}\left(p^{2} ; \mu\right)}+\mu=-p \cot \widetilde{\delta} \text {. }
$$

Expanding both sides of Eq. (5) in powers of $p^{2}$ and employing the effective range expansion Eq. (2), solutions for the running couplings $\widehat{C}_{2 n}(\mu)$ can be obtained. The first few are

$$
\begin{aligned}
\widehat{C}_{0}(\mu) & =-\frac{1}{\mu-1 / \widetilde{a}}, \\
\widehat{C}_{2}(\mu) & =\frac{1}{2} \widetilde{r}_{0}\left[\widehat{C}_{0}(\mu)\right]^{2}, \\
\widehat{C}_{4}(\mu) & =\frac{\left[\widehat{C}_{2}(\mu)\right]^{2}}{\widehat{C}_{0}(\mu)}+\widetilde{v}_{2}\left[\widehat{C}_{0}(\mu)\right]^{2} .
\end{aligned}
$$

Two important features of these solutions are:

\footnotetext{
${ }^{1}$ The $\widehat{C}$ couplings used throughout are a factor of $M / 4 \pi$ times the conventionally normalized $C$ 's found in the literature.
} 
- The EFT exhibits a nontrivial unstable infrared fixed point at $\mu \widehat{C}_{0}=-1$ and $\mu^{2 n+1} \widehat{C}_{2 n}=0$. This fixed point corresponds to a system with infinite scattering length, for which scattering near threshold due to $\widehat{C}_{0}$ looks scale invariant, in spite of the fact that the $\widehat{C}_{0}$ interaction is irrelevant by naive power counting $[7]$.

- Only for $\mu \sim \Lambda_{S}$ are the $\widehat{C}_{2 n}$ parameters of the size one would expect from naive power counting [2], assuming that the scattering parameters $\left\{\frac{1}{2} \widetilde{r}_{0}, \widetilde{v}_{n}\right\}$ of the short range interactions are of natural size; $\widehat{C}_{0}$ at this scale is fine tuned so that the system passes near the nontrivial infrared fixed point.

Although in the present example the PDS scheme allows for a formal summation of all the diagrams of the EFT, more realistic interactions require a systematic expansion which approximates the exact EFT result Eq. (田). We now explore two different expansion schemes.

\section{B. The Weinberg Expansion of the Effective Theory}

As discussed in 迥, the Weinberg approach [2 5] consists of expanding the kernel of the Lippmann-Schwinger equation, and then iterating it to all orders. The coefficients of the dimensionful contact interactions are taken to scale with $\Lambda_{S}$ according to their naive dimension, namely

$$
\widehat{C}_{2 n} p^{2 n} \sim\left(1 / \Lambda_{S}\right)\left(p / \Lambda_{S}\right)^{2 n}
$$

The kernel is then expanded in powers of $p / \Lambda_{S}$, where $p$ is the external momentum, so that counting powers of $p$ gives a way of organizing corrections to the potential. At $N^{t h}$ order in the expansion, the kernel is given by $V_{N}^{\mathrm{EFT}} \equiv \widehat{C}^{[N]}\left(p^{2} ; \mu\right)=\sum_{n=0}^{N} \widehat{C}_{2 n} p^{2 n}$.

Although traditionally the Weinberg approach is analyzed with a momentum cutoff, we can equally well use dimensional regularization with the PDS subtraction scheme [8]. At $N^{\text {th }}$ order in the expansion, the amplitude is given by Eq. (雨), with the exact interaction $\widehat{C}\left(p^{2} ; \mu\right)$ replaced by $\widehat{C}^{[N]}\left(p^{2} ; \mu\right)$. The $N$ couplings $\widehat{C}_{2 n}$ can be fixed by requiring that the theory correctly reproduce the first $N$ terms in the effective range expansion. The predictions for the phase shift $\widetilde{\delta}$ at leading order (LO), next-to-leading order (NLO), and next-to-nextto-leading order (NNLO) are then given by

$$
\begin{aligned}
(p \cot \widetilde{\delta})_{\mathrm{LO}} & =-\frac{1}{\widetilde{a}} ; \\
(p \cot \widetilde{\delta})_{\mathrm{NLO}} & =(p \cot \widetilde{\delta})_{\mathrm{LO}}+\frac{\frac{1}{2} \widetilde{r}_{0} p^{2}}{1+\frac{1}{2} \eta \widetilde{r}_{0} p^{2}} ; \\
(p \cot \widetilde{\delta})_{\mathrm{NNLO}} & =(p \cot \widetilde{\delta})_{\mathrm{NLO}}+\frac{p^{4}\left(\widetilde{v}_{2}+\frac{1}{4} \eta \widetilde{r}_{0}^{2}\right)}{\left(1+\frac{1}{2} \eta \widetilde{r}_{0} p^{2}\right)\left(1+\frac{1}{2} \eta \widetilde{r}_{0} p^{2}+\eta p^{4}\left[\widetilde{v}_{2}+\frac{1}{4} \eta \widetilde{r}_{0}^{2}\right]\right)}
\end{aligned}
$$

where $\eta \equiv(1 / \widetilde{a}-\mu)^{-1}$. The explicit $\mu$ dependence in the physical quantities is a result of neglecting counterterms necessary for renormalization at any finite order in the expansion.

For small $\mu$, these expressions fail above $p \sim\left(\widetilde{a} \widetilde{r}_{0}\right)^{-1 / 2}$ which is much below the expected EFT breakdown of $p \sim \Lambda_{S}$ (e.g., see the $\mu=0$ treatment in Ref. [12], or the equivalent 
momentum subtraction used in Ref. [2]). This failure is no surprise, since for small $\mu$ the $\widehat{C}_{2 n}$ couplings given in Eq. (6) do not obey the naive scaling assumed in Eq. (7), which was the starting point for Weinberg's expansion. Evidently, the assumed power counting is only obtained for $\mu \gtrsim \Lambda_{S}$, in which case we see that the result in Eq. (8) coincides with the effective range expansion up to higher order terms? in $p^{2} / \Lambda_{S}^{2}$.

\section{The KSW Expansion of the Effective Theory}

The KSW expansion starts from the assumption $\widetilde{a} \gg 1 / \Lambda_{S}$ and that the rest of the effective range parameters $\left\{\frac{1}{2} \widetilde{r}_{0}, \widetilde{v}_{n}\right\}$ scale with appropriate powers of $\Lambda_{S}$, according to their dimension. In this case, the amplitude Eq. (11) may be expanded as

$$
\mathcal{A}=-\frac{4 \pi / M}{(1 / \widetilde{a}+i p)}\left[1+\frac{\widetilde{r}_{0} / 2}{(1 / \widetilde{a}+i p)} p^{2}+\frac{\left(\widetilde{r}_{0} / 2\right)^{2}}{(1 / \widetilde{a}+i p)^{2}} p^{4}+\frac{\widetilde{v}_{2}}{(1 / \widetilde{a}+i p)} p^{4}+\ldots\right]
$$

provided one is not near the kinematic point corresponding to a pole in $\mathcal{A}$. This expansion can be realized in the EFT by applying the following power counting rules for $s$-wave interactions [8]:

1. Define a measure of small momenta called $Q$, and take $p, \mu$, and $1 / \widetilde{a}$ to be $\mathcal{O}(Q)$.

2. The $\widehat{C}_{2 n}(\mu)$ couplings, as given in Eq. (6), scale as $\mathcal{O}\left(Q^{-(n+1)}\right)$.

3. Loop momenta q are $\mathcal{O}(Q)$, while loop energies $q_{0}$ are $\mathcal{O}\left(Q^{2}\right)$. As a result, loop integrals (including two-nucleon propagators) scale as $\mathcal{O}(Q)$.

4. Derivative interactions contribute a power of $Q$ for each $\nabla$, and $Q^{2}$ for each $\partial_{t}$.

Summing up all Feynman diagrams to a given order in $Q$ and making use of Eq. (6) reproduces the expansion Eq. (9). Since this is a consistent expansion of a physical quantity, results are $\mu$-independent at each order. The most peculiar feature of the expansion is the scaling of $\widetilde{a}$ as $\mathcal{O}\left(Q^{-1}\right)$. This allows for an expansion in powers of $p / \Lambda_{S}$ while keeping powers of $p \widetilde{a}$ to all orders, but it will cause subtleties when the scale $m_{\pi}$ is introduced.

\section{ADDING TOY PIONS}

We now consider the more interesting case of an interaction with both long- and shortrange structure. The short-range interaction will remain unspecified for generality, giving the phase shift $\widetilde{\delta}$ when acting alone. For the long-distance part of the potential $V_{L}$, we choose the specific form of a delta-shell at a radius given by the pion Compton wavelength and a strength depending on $m_{\pi}$ in a manner consistent with chiral symmetry

\footnotetext{
${ }^{2}$ It is curious that in the limit $\mu \rightarrow \infty$, the Weinberg expansion to $N^{\text {th }}$ order exactly reproduces the first $N$ terms of the effective range expansion for $p \cot \widetilde{\delta}$.
} 


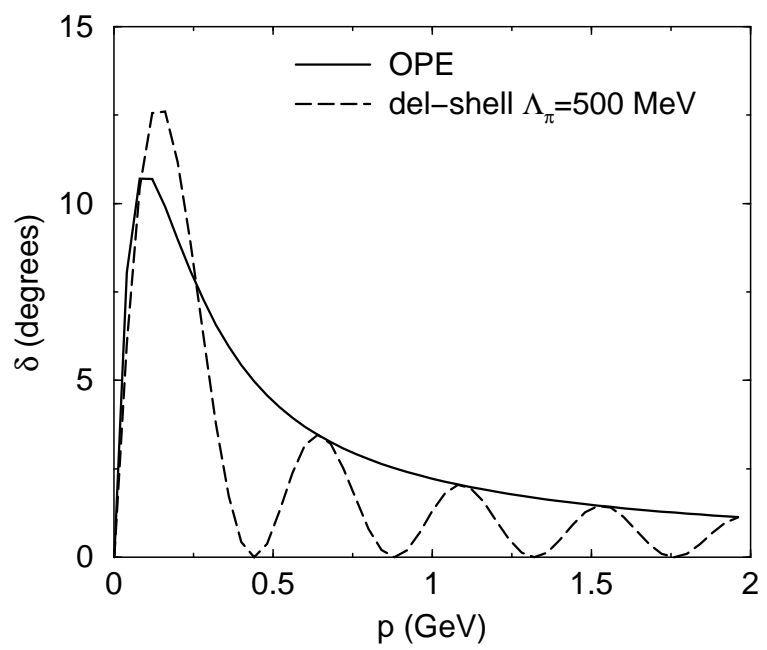

FIG. 1. By taking $\Lambda_{\pi}=500 \mathrm{MeV}$, the envelope of the OPE and delta-shell potential coincide.

$$
V_{L}(r)=-\frac{g_{\pi} m_{\pi}}{M} \delta\left(r-\frac{1}{m_{\pi}}\right)
$$

This caricature of the real one-pion exchange (OPE) potential allows us to analytically explore issues involving EFT expansions. The Yukawa part of the ${ }^{1} S_{0}$ channel OPE interaction in momentum space is

$$
\tilde{V}_{\mathrm{OPE}}=-\frac{4 \pi}{M \Lambda_{\mathrm{NN}}} \frac{m_{\pi}^{2}}{q^{2}+m_{\pi}^{2}}, \quad \Lambda_{\mathrm{NN}} \equiv \frac{16 \pi f_{\pi}^{2}}{g_{A}^{2} M} \simeq 300 \mathrm{MeV},
$$

where $m_{\pi}=140 \mathrm{MeV}$ is the pion mass, $f_{\pi}=93 \mathrm{MeV}$ is the pion decay constant, and $g_{A}=1.29$ is the axial coupling. The Fourier transform of $V_{L}$ is

$$
\tilde{V}_{L}=-\frac{4 \pi g_{\pi}}{M m_{\pi}} \frac{\sin q / m_{\pi}}{q / m_{\pi}}
$$

Comparison of the two expressions suggests that for $V_{L}$ to have comparable effects to $V_{\mathrm{OPE}}$, we should take $g_{\pi}=m_{\pi} / \Lambda_{\pi}$ with $\Lambda_{\pi} \sim \Lambda_{\mathrm{NN}}$. In fact, the properties of $V_{L}$ and the OPE Yukawa potential are most analogous if we take

$$
\Lambda_{\pi}=500 \mathrm{MeV} .
$$

By analogous, we mean that in both cases, the strength of the interaction is approximately $30 \%$ of the critical value needed to give a bound state (which occurs at $g_{\pi}=1$ in the toy model), and the strength of the phase shifts resulting from the two potentials are roughly comparable as shown in Fig. 11.

With $\Lambda_{S}>m_{\pi}$, it is straightforward to solve the Schrödinger equation

$$
\left[-\frac{\nabla^{2}}{M}+V_{S}+V_{L}\right] \psi=E \psi
$$

and determine the exact $s$-wave Feynman scattering amplitude $\mathcal{A}$ to be 


$$
\mathcal{A}=\mathcal{A}_{L}+\frac{4 \pi / M}{f(\xi)^{2}(p \cot \widetilde{\delta}-i p)-g_{\pi} m_{\pi} e^{2 i \xi} f(\xi)},
$$

with $\xi=p / m_{\pi}$ and

$$
f(\xi) \equiv 1-g_{\pi} \frac{e^{i \xi} \sin \xi}{\xi} .
$$

As in the previous section, $\widetilde{\delta}$ is the phase shift due to $V_{S}$ alone. The quantity $\mathcal{A}_{L}$ is the amplitude one finds for $V_{L}$ alone, when the short-range potential $V_{S}$ is turned off,

$$
\mathcal{A}_{L}=\frac{4 \pi g_{\pi}}{M m_{\pi}} \frac{\sin ^{2} \xi}{f(\xi) \xi^{2}} .
$$

The exact phase shift $\delta$ can be found to be

$$
\cot \delta=\frac{\left(\xi-g_{\pi} \sin \xi \cos \xi\right) \cot \widetilde{\delta}-g_{\pi} \cos ^{2} \xi}{g_{\pi} \sin ^{2} \xi \cot \widetilde{\delta}+\xi+g_{\pi} \sin \xi \cos \xi} .
$$

From this, one can determine the exact effective range parameters defined by

$$
p \cot \delta=-\frac{1}{a}+\frac{1}{2} r_{0} p^{2}+\sum_{n=2}^{\infty} v_{n} p^{2 n}+\ldots
$$

in terms of the short-distance effective range parameters Eq. (2). For example, with $g_{\pi} \equiv$ $m_{\pi} / \Lambda_{\pi}$,

$$
\begin{aligned}
a & =\frac{1-\widetilde{a}\left(\Lambda_{\pi}+m_{\pi}\right)}{m_{\pi}\left(1-\widetilde{a} m_{\pi}\right)-\Lambda_{\pi}}, \\
r_{0} & =\frac{2 m_{\pi}\left(1-\widetilde{a} m_{\pi}\right)^{2}+2 \Lambda_{\pi}\left(1-3 \widetilde{a} m_{\pi}\right)\left(1-\widetilde{a} m_{\pi}\right)+3 \widetilde{a}^{2} \Lambda_{\pi}^{2} m_{\pi}^{2} \widetilde{r}_{0}}{3 m_{\pi}^{2}\left[1-\widetilde{a}\left(\Lambda_{\pi}+m_{\pi}\right)\right]^{2}} .
\end{aligned}
$$

We wish to reproduce the full amplitude $\mathcal{A}$ in Eq. (15) in a systematic expansion of an EFT, replacing the potential $V_{S}$ with contact interactions. We first show that the Feynman amplitude can be exactly reproduced by the effective theory, as was the case when we considered $V_{S}$ alone. Subsequently, we address the issue of how to compute these amplitudes in perturbation theory, which is of practical interest for realistic $N N$ scattering, where exact analytical solutions to the EFT are impossible to obtain.

\section{A. The Exact Solution of the Effective Theory with Toy Pions}

Taking an EFT with contact interactions $\widehat{C}\left(p^{2} ; \mu\right)$ and the explicit long-range potential $V_{L}$ given in Eq. (10), it is again possible to sum up all diagrams using dimensional regularization and the PDS subtraction scheme (see Appendix A) resulting in

$$
\mathcal{A}^{\mathrm{EFT}}=\mathcal{A}_{L}+\frac{4 \pi / M}{-f(\xi)^{2}\left[\widehat{C}^{-1}\left(p^{2} ; \mu\right)+\mu+i p\right]-g_{\pi} m_{\pi} e^{2 i \xi} f(\xi)},
$$


with $f(\xi)$ and $\mathcal{A}_{L}$ as in Eqs. (16, 17). A comparison between the above expression and the exact amplitude Eq. (15) shows that the EFT reproduces the full theory provided the $\widehat{C}$ couplings are chosen to satisfy

$$
\frac{1}{\widehat{C}\left(p^{2} ; \mu\right)}+\mu=-p \cot \widetilde{\delta} .
$$

This is exactly the same result that we derived earlier in the theory without long-range interactions, Eq. (5). Thus, the $\widehat{C}_{2 n}$ interactions truly represent the short-distance physics alone, and are independent of $V_{L}$. (This need not in general be true for the more realistic case of nonzero overlap in space between $V_{S}$ and $V_{L}$.)

\section{B. The Weinberg Expansion with Toy Pions}

In Weinberg's power counting scheme, the scale $\Lambda_{\pi}$ is considered a short-distance scale and so insertions of $V_{L}$ are of the same order as insertions of $\widehat{C}_{0}$, as seen from Eq. (12). Thus to $N^{\text {th }}$ order in the expansion, the effective potential is given byf

$$
V_{N}^{\mathrm{EFT}}=V_{L}+\sum_{n=0}^{N} \widehat{C}_{2 n} p^{2 n} .
$$

The $N^{\text {th }}$ order expression for the Feynman amplitude is just given by the exact EFT solution Eq. (22) with $\widehat{C}\left(p^{2} ; \mu\right)$ replaced by the truncated series of $\widehat{C}_{2 n} p^{2 n}$ in Eq. (24). The discussion now is entirely analogous to that of $\$$ IIB. Again the amplitude is not renormalized and therefore is $\mu$-dependent. However, as long as $\mu \gtrsim \Lambda_{S}$ and the $N$ couplings $\widehat{C}_{2 n}$ are chosen to reproduce the effective range expansion (up to higher order terms), the exact expression for the amplitude Eq. (15) is obtained with $p \cot \widetilde{\delta}$ replaced by the first $N$ terms in the short-distance effective range expansion. Note that Weinberg's expansion is functionally equivalent to the modified effective range expansion [13] as long as the overlap between $V_{L}$ and $V_{S}$ is properly accounted for, as discussed in Ref. [14].

\section{The KSW Expansion with Toy Pions}

\section{The Naive KSW Expansion and Its Failings Near the Pole}

Once pions are included in the EFT, the KSW expansion treats $m_{\pi}$ as order $Q$ as well, generalizing the rules of $₫ \amalg \mathrm{IO}$. This is consistent with chiral perturbation theory, which treats $p$ and $m_{\pi}$ to be of the same order and small. It follows that $V_{L}$ in Eq. (12) is $\mathcal{O}\left(Q^{0}\right)$, which is subleading compared to $\widehat{C}_{0}$, which is $\mathcal{O}\left(Q^{-1}\right)$. Therefore, pions enter the amplitude perturbatively [8], unlike in the Weinberg approach. It is advantageous to treat the OPE potential perturbatively, since perturbative long-range interactions are analytically

\footnotetext{
${ }^{3}$ In general, these operators have to be specified more precisely, for example $\widehat{C}_{2} p^{2}$ must be replaced by $\frac{1}{2} \widehat{C}_{2}\left\{\hat{p}^{2}, \delta^{3}(\hat{r})\right\}$ and so on.
} 
tractable, at least up to NNLO. While this is not a major benefit in two-nucleon systems, it is likely to be very valuable in many-body problems due to computational simplifications.

However, there is a subtlety associated with implementing this method, having to do with the large scattering length which arises from the delicate interplay between short-range physics and pion exchange. Making use of the exact solution for $\widehat{C}_{0}$ from Eq. (6), one finds that the leading contribution to the amplitude at $\mathcal{O}\left(Q^{-1}\right)$ is

$$
\mathcal{A}_{-1}^{\mathrm{EFT}}=-\frac{4 \pi / M}{1 / \widetilde{a}+i p} .
$$

This has a pole at $p_{\star}=i / \widetilde{a}$, in contrast to the exact amplitude which has a pole at $p_{\star} \simeq i / a$. Even if $1 / a$ and $1 / \widetilde{a}$ differ by an amount small compared to $m_{\pi}$, since the threshold cross section is $4 \pi a^{2}$, this expansion will fail to accurately describe low-momentum scattering if either $a$ or $\widetilde{a}$ are particularly large. For example, in the extreme case where the scattering length $a$ is infinite, then the true amplitude at threshold is also infinite. However, $\widetilde{a}$ would be finite in that case, and so Eq. (25) would require the subleading perturbative corrections to the cross section to be infinite in order to produce the correct result!

The situation is similar to the problem of calculating $e^{+} e^{-}$scattering amplitudes at the $Z^{0}$ pole in perturbation theory. Even though the true value for the $Z^{0}$ mass and the tree level value only differ by an $\mathcal{O}(\alpha)$ contribution, practical calculations are done by perturbing around the exact $Z^{0}$ mass and introducing a mass shift at each order in perturbation theory to cancel the subsequent radiative corrections.

Similarly, in the present example, a small difference between $1 / \widetilde{a}$ and $-i p_{\star}$ does not ensure an accurate description of scattering for $p \sim|1 / a|$. For large and positive $a$, where a shallow bound state exists and the pole in $\mathcal{A}$ is on the physical sheet (such as the deuteron pole in ${ }^{3} S_{1} N N$ scattering), an expansion around the true location of the pole is necessary. For large and negative $a$, where the pole is kinematically inaccessible (as in the ${ }^{1} S_{0}$ channel), it suffices to reproduce the exact scattering length at leading order. This is the procedure of matching at LO already carried out in the literature [B], but it needed to be understood from the perspective of the short-distance scales given in Eq. (6), because extending the procedure to NLO differs from the conventional approach. The following discussion has much in common with that of Mehen and Stewart [15] and Rupak and Shoresh [16].

\section{The Pole Expansion}

The resolution, as in the example of the $Z^{0}$ pole, is to reorder the expansion, making use of the fact that the difference between $1 / \widetilde{a}$ and either $1 / a$ or $-i p_{\star}$ is $\mathcal{O}\left(Q^{2}\right)$. For the case relevant to $N N$ scattering in the ${ }^{1} S_{0}$ channel, we write

$$
\frac{1}{\widetilde{a}}=\frac{1}{a}+\sum_{k=2}^{\infty} \alpha_{k}
$$

where the $\alpha_{k}$ are $\mathcal{O}\left(Q^{k}\right)$ functions of $a, m_{\pi}, \Lambda_{\pi}$ and $\mu$. For example, the long-range potential we are considering gives

$$
\frac{1}{\widetilde{a}}=\frac{\Lambda_{\pi}+m_{\pi}-a m_{\pi}^{2}}{1+a \Lambda_{\pi}-a m_{\pi}} \quad \Longrightarrow \quad \alpha_{n}=-\Lambda_{\pi}\left(\frac{a m_{\pi}-1}{a \Lambda_{\pi}}\right)^{n} .
$$


Substituting the expression for the short-distance scattering length Eq. (26) into $\widehat{C}_{0}$ from Eq. (6) gives an expansion in $Q$ :

$$
\widehat{C}_{0}(\mu) \equiv \sum_{k=-1}^{\infty} \widehat{C}_{0}^{[k]}(\mu),
$$

where $\widehat{C}_{0}^{[k]}$ is of order $Q^{k}$. The first three contributions are

$$
\widehat{C}_{0}^{[-1]}=-\frac{1}{\mu-1 / a}, \quad \widehat{C}_{0}^{[0]}=-\alpha_{2}\left(\widehat{C}_{0}^{[-1]}\right)^{2}, \quad \widehat{C}_{0}^{[1]}=\alpha_{2}^{2}\left(\widehat{C}_{0}^{[-1]}\right)^{3}-\alpha_{3}\left(\widehat{C}_{0}^{[-1]}\right)^{2} .
$$

This expansion ensures that the EFT yields the exact scattering length $a$ at each order in the KSW expansion, with $\widehat{C}_{0}^{[k]}$ corrections to cancel the perturbative contributions of the pions arising at each order. Note that $\widehat{C}_{0}$ as defined in Eq. (28) remains $m_{\pi}$-independent, even though the $\widehat{C}_{0}^{[k]}$ terms each have a rather complicated $m_{\pi}$ dependence.

Once $\widehat{C}_{0}$ is expanded, the exact solutions for the $\widehat{C}$ couplings in Eq. (6) imply that each $\widehat{C}_{2 n}$ coefficient must be similarly expanded. For instance,

$$
\begin{array}{ll}
\widehat{C}_{2}^{[-2]}=\frac{1}{2} \widetilde{r}_{0}\left(\widehat{C}_{0}^{[-1]}\right)^{2}, \quad \widehat{C}_{2}^{[-1]}=\widetilde{r}_{0}\left(\widehat{C}_{0}^{[-1]} \widehat{C}_{0}^{[0]}\right), \quad \ldots, \\
\widehat{C}_{4}^{[-3]}=\frac{\left(\widehat{C}_{2}^{[-2]}\right)^{2}}{\widehat{C}_{0}^{[-1]}}, & \widehat{C}_{4}^{[-2]}=2 \frac{\widehat{C}_{2}^{[-1]} \widehat{C}_{2}^{[-2]}}{\widehat{C}_{0}^{[-1]}}+\alpha_{2}\left(\widehat{C}_{2}^{[-2]}\right)^{2}+\widetilde{v}_{2}\left(\widehat{C}_{0}^{[-1]}\right)^{2}, \ldots .
\end{array}
$$

That the leading behavior of each $\widehat{C}_{2 n}$ is determined by lower dimensional operators is further evidence of the EFT being tuned to lie near a nontrivial fixed point.

Note that in the KSW expansion, the parameter $\widetilde{r}_{0}$ first enters at $\mathcal{O}\left(Q^{0}\right)$ through an insertion of $\widehat{C}_{2}^{[-2]} p^{2}$, while the next new parameter $\widetilde{v}_{2}$ does not enter until $\mathcal{O}\left(Q^{2}\right)$ through an insertion of $\widehat{C}_{4}^{[-2]} p^{4}$. In general, the short-distance effective range parameters Eq. (21) enter the expansion of $\mathcal{A}$ through the operators $\widehat{C}_{2 n}^{[-2]} p^{2 n} \sim \mathcal{O}\left(Q^{2 n-2}\right)$ for each $n \geq 1$. Thus in the KSW expansion, one new parameter is encountered for every two powers of $Q$. The NNLO amplitude $\mathcal{A}_{1}$, for example, involves graphs not included in $\mathcal{A}_{0}$; nevertheless, it is completely parameterized by the same two numbers $a$ and $\widetilde{r}_{0}$. The fact that new parameters appear in the expansion of $\mathcal{A}$ with only even powers of $Q$ occurs because the effective range expansion of $p \cot \widetilde{\delta}$ is in even powers of momentum. A valid fitting procedure for the EFT should reflect this behavior, with one free parameter at LO to fix the scattering length $a$, one free parameter at NLO to fix $\widetilde{r}_{0}$, and subsequent free parameters appearing at every other order $\mathcal{O}\left(Q^{2 n-2}\right)$ to fix each $\widetilde{v}_{n \geq 2}$.

\section{A General Algorithm for Fixing Coupling Constants}

The Weinberg expansion has a relatively simple algorithm for fixing the low-energy constants of the EFT: Taylor expand the EFT result for $p \cot \delta$ and match to the effective range expansion at each order (making sure the long-distance effects are properly taken into account [14]). The procedure to fix the low-energy constants in the KSW expansion is more obscure. When the nucleon interactions happen to be fine-tuned, high order perturbative 
pion contributions can in principle have relatively large effects on low-energy observables due to cancellations at lower orders in the expansion. Our simple long-distance potential $V_{L}$ allows for an analytical determination of all the $\vec{C}_{2 n}^{[k]}$ couplings (as we will show in an example in $\S[\mathrm{IV})$. However, for realistic situations one needs an algorithm for fixing these couplings. The above discussion makes it possible to surmise a general prescription:?

1. Expand each $\widehat{C}_{2 n}$ coupling as

$$
\widehat{C}_{2 n}=\sum_{k=-(n+1)}^{\infty} \widehat{C}_{2 n}^{[k]}
$$

where $\widehat{C}_{2 n}^{[k]}$ is $\mathcal{O}\left(Q^{k}\right)$ in the expansion, and compute the amplitude to the desired order.

2. Use the threshold amplitude to fix $\widehat{C}_{0}^{[-1]}$ so that the LO result reproduces the experimental scattering length $a$, and fix the higher order $\widehat{C}_{0}^{[k]}$ by requiring the scattering length be unchanged at each higher order.

3. Determine the renormalization group equations in the PDS scheme for the $\widehat{C}_{2 n}$ couplings to the order one is working. The beta function for $n \geq 1$ is always of the form

$$
\mu \frac{d \widehat{C}_{2 n}}{d \mu}=2 \mu \widehat{C}_{0} \widehat{C}_{2 n}+\mu \frac{d F_{2 n}}{d \mu}
$$

where $F_{2 n}$ only depends on couplings $\widehat{C}_{2 m}$ with $m<n$ and possibly the long-distance physics.] Note that for $n>1$, the $F_{2 n}$ are actually the leading contribution in the $Q$-expansion of Eq. (31). Solving this equation leads to

$$
\widehat{C}_{2 n}=\widetilde{v}_{n} \widehat{C}_{0}^{2}+F_{2 n},
$$

where $\widetilde{v}_{n}$ is an undetermined constant of integration treated as $\mathcal{O}\left(Q^{0}\right)$. This solution should then be expanded in $Q$ to produce each of the $\widehat{C}_{2 n}^{[k]}$, s to be used in the calculated amplitude $\mathcal{A}$.

\footnotetext{
${ }^{4}$ For the toy models we are considering, the Weinberg expansion does not suffer from this problem, since the long-range interactions are included completely at lowest order. However, for real $N N$ scattering, the Weinberg method also involves a chiral expansion of the long-range interactions beyond OPE, and so in principle subleading contributions could similarly exhibit large effects on low-energy scattering, complicating the determination of the contact interactions $\widehat{C}_{2 n}$.

${ }^{5}$ Here we describe the procedure for a system without a bound state, such as $N N$ scattering in the ${ }^{1} S_{0}$ channel. Systems with a bound state, where one needs to fix the pole $i p_{\star}$ at LO instead of the scattering length $a$, are considered elsewhere [17,16]. Our procedure can easily be extended to these cases.

${ }^{6}$ The toy pions Eq. (10) are well behaved at the origin and therefore never contribute to the $F_{2 n}$ 's. Actual pion exchange has a finite overlap with the EFT contact interactions, so $\log \mu$ terms could occur. However, at least to NNLO in the $Q$-expansion, only $F_{0}$ has any such contribution.
} 
4. Within the $Q$-expansion, the phase shift is computed consistently from this amplitude $\mathcal{A}$ in terms of the parameters $\left\{\frac{1}{2} \widetilde{r}_{0}, \widetilde{v}_{n}\right\}$, which are the short-distance effective range parameters. These parameters are then determined by finding the best fit to the data with an appropriate weighting in momentum (as discussed in $\S[\bar{D})$.

In this manner, contact is made between the plethora of terms in the KSW expansion, and the effective range parameters $\left\{\frac{1}{2} \widetilde{r}_{0}, \widetilde{v}_{n}\right\}$ characterizing the short distance physics. At a given order, the KSW and Weinberg expansions have the same number of free parameters to fit. This general algorithm will correctly reproduce the KSW expansion in the presence of pions as explicitly shown in $\S \mathbb{\square}$.

Note that additional contact interactions with quark mass insertions are possible [8], such as $D_{2} m_{\pi}^{2}$ at NLO and $D_{22} m_{\pi}^{2} p^{2}$ at NNLO. However, to distinguish between these operators and the $C_{2 n}^{[k]}$ interactions would require additional information beyond $N N$ scattering, such as $\pi d$ scattering. Therefore, in the present discussion we subsume them into our definitions of the $C_{2 n}^{[k]}$ s.

\section{EXAMPLES}

We now illustrate the above procedure for fixing coupling constants in the KSW expansion scheme by three examples. Choosing an explicit form for the short-distance potential allows us to check that this method is actually working. In all cases, we will tune the couplings in the model to give $a=-23 \mathrm{fm}$ similar to the ${ }^{1} S_{0}$ channel of $N N$ scattering.

\section{A. The Toy Pion with a Short-range Delta-shell Interaction}

Our first example will utilize the toy pions of Eq. (10). We choose the short-distance physics to also be modeled by a delta-shell so an analytic solution is possible

$$
V=V_{S}+V_{L}, \quad V_{S}(r)=-g_{\rho} \frac{m_{\rho}}{M} \delta\left(r-\frac{1}{m_{\rho}}\right), \quad V_{L}(r)=-\frac{m_{\pi}^{2}}{M \Lambda_{\pi}} \delta\left(r-\frac{1}{m_{\pi}}\right),
$$

with $\Lambda_{\pi}=500 \mathrm{MeV}$. The exact phase shift is then just given by Eq. (18) with

$$
p \cot \widetilde{\delta}=\frac{\xi_{\rho}-g_{\rho} \sin \xi_{\rho} \cos \xi_{\rho}}{g_{\rho} \sin ^{2} \xi_{\rho}},
$$

and $\xi_{\rho}=p / m_{\rho}$. Taking $m_{\rho}=770 \mathrm{MeV}$, we choose $g_{\rho}=0.915$ to give the scattering length $a=-23 \mathrm{fm}$.

The EFT amplitude $\mathcal{A}$ up to NNLO is quoted in Appendix B. The contact interactions $\widehat{C}_{2 n}^{[k]}$ were worked out in general in Eqs. (29, 30), so all that is left to do is implement the procedure outlined in $\S[\Pi \mathrm{C}$. 3 to fix these constants. The EFT calculation at LO only depends on $\widehat{C}_{0}^{[-1]}$, which as seen from Eq. (29) is fixed by the experimental scattering length.

At NLO, pions begin to contribute, and the perturbative correction $\widehat{C}_{0}^{[0]}$ is chosen to ensure the scattering length does not shift. This specifies $\alpha_{2}$, which coincides with the analytic result given in Eq. (27). The contact interaction $\widehat{C}_{2}^{[-2]}$ also contributes at this order and depends on $\widetilde{r}_{0}$. We use its exact value, which can be calculated from Eq. (34) 

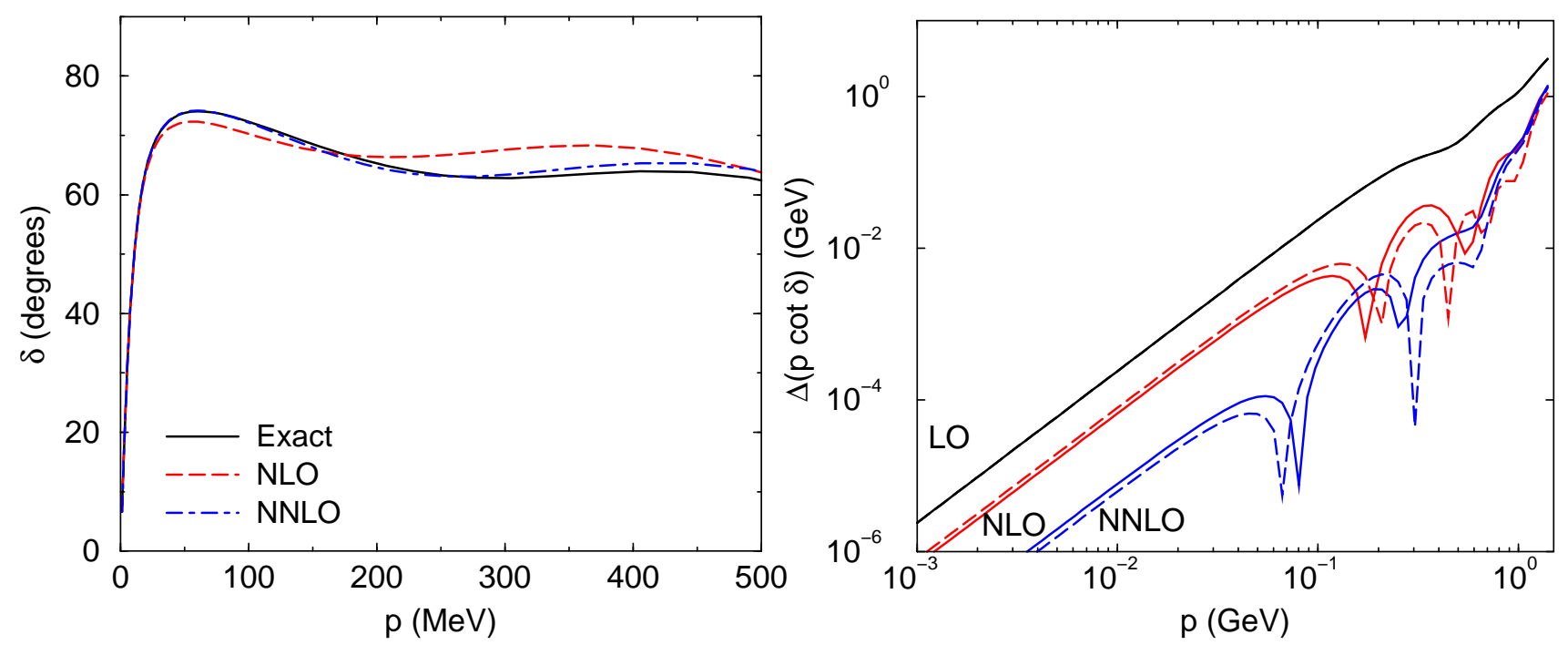

FIG. 2. The algorithm of $₫[I I C 3$ for the KSW expansion applied to the two delta-shell model. The left plot shows that as more orders are added to the EFT calculation, the agreement with the exact phase shift improves. The right plot shows the corresponding errors in the observable $p \cot \delta$.

$$
\widetilde{r}_{0}=\frac{2\left(1+g_{\rho}\right)}{3 g_{\rho} m_{\rho}}=0.36 \mathrm{fm},
$$

to test our fit. Afterwards, we will discuss how to find this value when the short-distance physics is not known a priori.

At NNLO, choosing $\widehat{C}_{0}^{[1]}$ so the scattering length does not shift produces $\alpha_{3}$ as given by Eq. (27). The other new contact interactions $\widehat{C}_{2}^{[-1]}$ and $\widehat{C}_{4}^{[-3]}$ are fully determined by already specified quantities Eq. (30), so there are no new constants to fix at this order as already discussed.

The results are shown in Fig. 2. On the left, the phase shift as a function of momentum for the exact result (solid line) is compared to the EFT calculation at NLO (dashed) and NNLO (dot-dashed). Note that the results improve as more orders are added, even when there are no additional free parameters (as occurs at NNLO). The right plot of Fig. 2 shows the improvement in the observable $p \cot \delta$ by plotting its error at each order (solid lines).

If the exact value for $\widetilde{r}_{0}$ were not known, as is the case in real data, we could treat $\widetilde{r}_{0}$ as a free parameter of the effective theory, varying its value to achieve the best global fit to data over a representative momentum range such as $\left[1 / a, \Lambda_{\pi}\right]$. Doing this at both NLO and NNLO produces the dashed lines in the right plot of Fig. 2, coinciding quite well with the exact result. Another indication of this agreement can be found by comparing the fit values for $\widetilde{r}_{0}$ with the exact value Eq. (35). The EFT with a global fit gives $\widetilde{r}_{0}=0.41 \mathrm{fm}$ at $\mathrm{NLO}$ and $\widetilde{r}_{0}=0.37 \mathrm{fm}$ at NNLO, showing convergence to the exact result $\widetilde{r}_{0}=0.36 \mathrm{fm}$ of Eq. (35), differing only by higher order contributions which the fit cannot resolve.

The point at which this fitting procedure breaks down can also be determined. The EFT should only work for momentum below a scale associated with underlying physics not explicitly accounted for in the Lagrangian, which is $m_{\rho}$ for this two delta-shell model. Up to this point, we have computed the phase shift by expanding the expression [Q] 


$$
\begin{aligned}
\delta & =\frac{1}{2 i} \ln \left[1+i \frac{M p}{2 \pi}\left(\mathcal{A}_{-1}+\mathcal{A}_{0}+\ldots\right)\right] \\
& \simeq \frac{1}{2 i} \ln \left(1+i \frac{M p}{2 \pi} \mathcal{A}_{-1}\right)+\frac{M p}{4 \pi}\left(\frac{\mathcal{A}_{0}}{1+i \frac{M p}{2 \pi} \mathcal{A}_{-1}}\right)+\ldots,
\end{aligned}
$$

but we could also have kept the full expression Eq. (36). These two expressions are equivalent up to terms that are higher order in the expansion. Those extra terms are an estimate of the corrections to the actual result and depend on the radius of convergence. The breakdown of the KSW expansion can be identified as the point at which the two expressions Eqs. (36, 37) diverge from each other. Doing this exercise for the two delta-shell model using the results of the fit to NLO and NNLO reveals the breakdown to be near $m_{\rho}$ as expected [5].

The delta-shell model for the pion allows us to obtain analytical expressions for the scattering amplitude and to check the algorithm for determining the $\widehat{C}_{2 n}^{[k]}$ coefficients of the EFT as described in $\S$ IIC 3 . However, this toy model can be criticized as being too simple to provide evidence that the KSW expansion will work for actual $N N$ scattering. We therefore consider models where pion exchange is represented by the correct OPE potential for the ${ }^{1} S_{0}$ channel.

\section{B. The Two-Yukawa Model}

We now take a more sophisticated model with a long-distance potential given by the Yukawa part of the ${ }^{1} S_{0}$ OPE potential, and a short-distance Yukawa potential characterized by the rho mass

$$
V=V_{\pi}+V_{S}, \quad V_{\pi}=-\alpha_{\pi} \frac{e^{-m_{\pi} r}}{r}, \quad V_{S}=-\alpha_{\rho} \frac{e^{-m_{\rho} r}}{r} .
$$

The pion coupling is taken to be $\alpha_{\pi}=g_{A}^{2} m_{\pi}^{2} /\left(16 \pi f_{\pi}^{2}\right) \simeq 0.075$, as in the real world, and the rho coupling $\alpha_{\rho}=1.05$ is tuned to give a large scattering length $a=-23 \mathrm{fm}$, as observed in data for the ${ }^{1} S_{0}$ partial wave of $N N$ scattering. Analyzing scattering from the short-range $V_{S}$ potential alone then yields

$$
\widetilde{a}=-1.17 \mathrm{fm}, \quad \widetilde{r}_{0}=0.765 \mathrm{fm},
$$

and the effective range expansion for the phase shift $\widetilde{\delta}$ works well up to $p \simeq m_{\rho} / 2$, as expected from analyticity considerations.

The EFT amplitude with potential pions has been calculated up to NNLO in Refs. 16,18. We make use of that amplitude and fix the contact interactions according to the algorithm described in $\S$ [IIC 3. This gives a LO amplitude that depends upon $a$, and NLO and NNLO amplitudes that also depend upon $\widetilde{r}_{0}$, the short-distance effective range parameter. The results obtained for the actual value $\widetilde{r}_{0}=0.765 \mathrm{fm}$ are shown in Fig. 3 for NLO and NNLO. The corresponding values for the full effective range are $r_{0}=2.1 \mathrm{fm}$ and $r_{0}=1.3 \mathrm{fm}$ respectively, compared to the exact value $1.6 \mathrm{fm}$.

The result from using the Weinberg approach (short dashed line) is also shown. Since it is fit at low-momentum, the agreement there is better than the KSW expansion. Both expansion schemes coincide at large momentum, which is in agreement with the idea that the Weinberg approach includes what the KSW expansion considers to be subleading terms. 

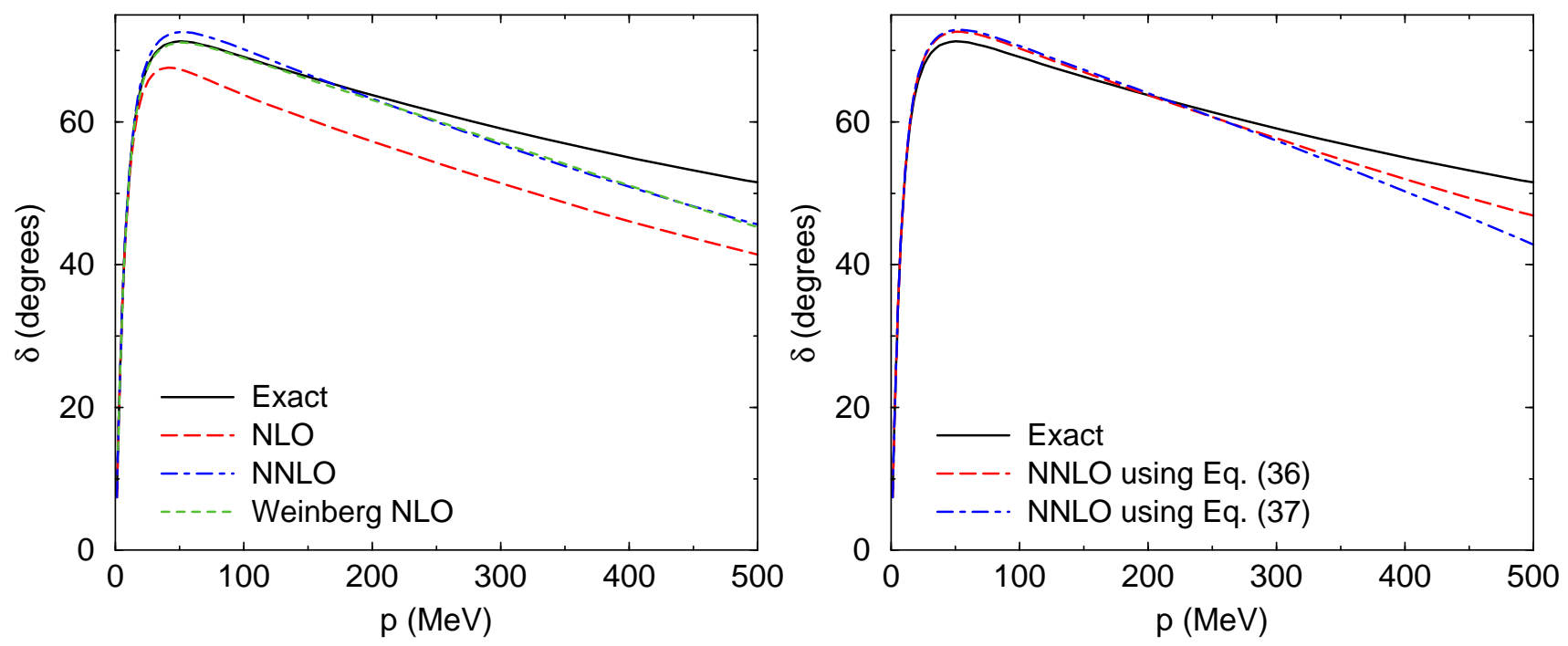

FIG. 3. The algorithm of $\oint$ [IC 3 for the KSW expansion applied to the two-Yukawa model. The left plot compares NLO and NNLO results from the EFT to the exact result. The right plot shows the NNLO results using the different expansion schemes Eqs. (36, 37) to determine the breakdown scale, which appears to be around $400 \mathrm{MeV}$.

The right plot of Fig. 3 has a comparison of the NNLO result for the phase shift, as determined from Eqs. (36, 37), suggesting that the higher order corrections to the expansion become important at a scale around $p \sim 400 \mathrm{MeV}$. That the breakdown is a factor of two smaller than for a delta-shell "rho" is in agreement with the analysis of the two-Yukawa model in Ref. [14.

Instead of using the actual value for $\widetilde{r}_{0}$, we could also treat it as a free parameter and use a one-parameter global fit to data. This gives $\widetilde{r}_{0}=0.400 \mathrm{fm}$ at NLO and $\widetilde{r}_{0}=0.671 \mathrm{fm}$ at NNLO. The deviations from the true value of $\widetilde{r}_{0}$ by $47 \%$ and $12 \%$ respectively are roughly what is expected from the estimates $\mathcal{O}\left(\alpha_{\pi} / \alpha_{\text {crit }}\right)$ and $\mathcal{O}\left(\left[\alpha_{\pi} / \alpha_{\text {crit }}\right]^{2}\right)$, where $\alpha_{\text {crit }} \simeq 0.25$ is the critical pion coupling that would lead to a bound state in the OPE potential.

Note that a low-momentum fit will give a more accurate result at low-momentum [16], but overall only differ from our approach by higher order terms. The result will be the same breakdown scale as we observe in Fig. 3. However, it is important to realize at NNLO no new parameter is required in our way of fitting, in contrast to the method employed in Ref. [16], so that our fit has one fewer free parameters.

\section{The Three-Yukawa Model}

The two-Yukawa model is unable to reproduce the large effective range $r_{0}=2.63 \mathrm{fm}$ of the ${ }^{1} S_{0}$ channel for any choice of couplings in Eq. (38). So as a final example, we add one more Yukawa interaction in order to have the proper effective range. We take for our potential

$$
V=V_{\pi}+V_{S}, \quad V_{\pi}=-\alpha_{\pi} \frac{e^{-m_{\pi} r}}{r}, \quad V_{S}=-\alpha_{\sigma} \frac{e^{-m_{\sigma} r}}{r}+\alpha_{\rho} \frac{e^{-m_{\rho} r}}{r},
$$




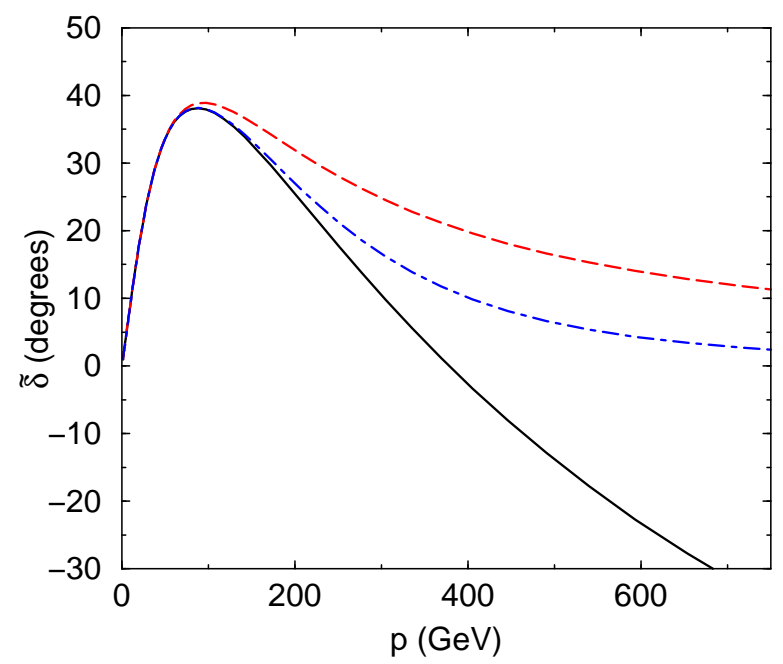

FIG. 4. The phase shift $\widetilde{\delta}$ due to scattering from the combined $\rho$ and $\sigma$ contributions to the potential $V(r)$ in Eq. (40). The exact result (solid) is compared with the effective range expansion to order $p^{2}$ (dashed) and $p^{4}$ (dot-dashed).

with the physical values for $m_{\pi}, m_{\rho}$, and $\alpha_{\pi}$, and with $m_{\sigma}=500 \mathrm{MeV}$. We then determine the final two parameters in order to obtain $a=-23 \mathrm{fm}$ and $r_{0}=2.6 \mathrm{fm}$, yielding $\alpha_{\sigma}=7$ and $\alpha_{\rho}=14.65$. This three-Yukawa model is reminiscent of the Bonn potential, which is known to model the data well.

Examining scattering from the short-range potential $V_{S}$ alone, we find

$$
\widetilde{a}=-3.3 \mathrm{fm}, \quad \widetilde{r}_{0}=2.59 \mathrm{fm},
$$

which immediately suggests that we will encounter serious problems due to the large size of $\widetilde{r}_{0}$. Recall that $\widetilde{r}_{0}$ is assumed to be $\mathcal{O}\left(1 / \Lambda_{S}\right)$ in all effective field theory approaches proposed to date. For this model, however, the scale $\Lambda_{S}$ corresponds to $m_{\sigma} / 2=250 \mathrm{MeV}$, whereas $\widetilde{r}_{0} \simeq 1 /(80 \mathrm{MeV})$ corresponds to a much smaller scale.

Performing an effective range expansion for $\widetilde{\delta}$, we obtain the result shown in Fig. $甘$. The short-distance effective range expansion, if carried to high enough order, does indeed have a radius of convergence set by $p \sim m_{\sigma} / 2$. However, the expansion at $\mathcal{O}\left(p^{2}\right)$ deviates from the exact result already at momenta below $m_{\pi}$. This suggests that an EFT expansion to the same order will also fail at momenta below the pion mass, even with the pion included explicitly.

Applying the EFT to the three-Yukawa model reveals this to be the case. The results for NLO and NNLO using the exact $\widetilde{r}_{0}=2.59 \mathrm{fm}$ are shown in Fig. 0 . The EFT with a global fit instead gives $\widetilde{r}_{0}=1.68 \mathrm{fm}$ at NLO and $\widetilde{r}_{0}=3.71 \mathrm{fm}$ at NNLO, also showing no signs of convergence. Similarly, the full effective range gives $r_{0}=3.7 \mathrm{fm}$ at NLO and $r_{0}=1.1 \mathrm{fm}$ at NNLO, both equally far from the exact value $2.6 \mathrm{fm}$.

The Weinberg approach result does better overall, only deviating from the exact result around $300 \mathrm{MeV}$. This indicates that the subleading order terms which are accounted for in this case are larger than anticipated from the KSW power counting. At best, the breakdown scale for the KSW power counting appears also to be around $300 \mathrm{MeV}$ as seen in the right 

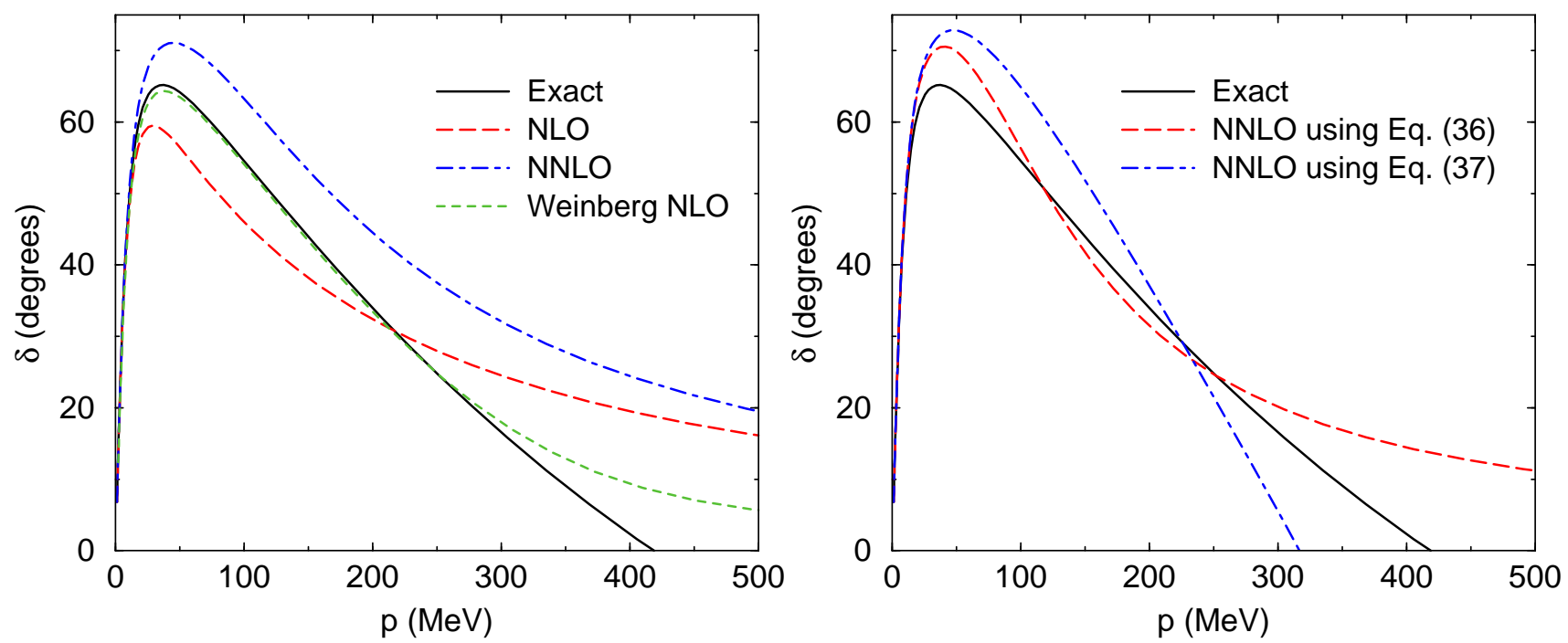

FIG. 5. The algorithm of $\S$ IIC 3 for the KSW expansion applied to the three-Yukawa model. The right plot shows the NNLO results using the different expansion schemes Eqs. (36, 37) to determine the breakdown scale.

plot of Fig. 月. If the $\sigma$ meson can be described accurately by non-irreducible two-pion effects, the Weinberg counting would include this at NLO and could possibly improve the radius of convergence even further [19].

\section{Lessons Learned}

The point of this section was to test the efficacy of the expansion algorithm of §IIIC3. An evident trend can be spotted: the larger the parameter $\widetilde{r}_{0}$, the slower the expansion converges, verifying the claims of Cohen and Hansen [20] and discussed by Cohen 21] that the short distance physics in $N N$ scattering may not look sufficiently short. In the first model, with both long- and short-range delta-shell potentials, $\widetilde{r}_{0}=0.36 \mathrm{fm}$ and the convergence is excellent. The second example includes a realistic OPE potential and an attractive short-range Yukawa, giving $\widetilde{r}_{0}=0.77 \mathrm{fm}$. Here too, the NNLO calculation does quite well, although not as good as in the first example. The third example with threeYukawa interactions has an enormous $\widetilde{r}_{0}=2.6 \mathrm{fm}$, and the expansion does poorly at NNLO. Note that in the EFT, the only difference between the two- and three-Yukawa models is the value for $\widetilde{r}_{0}$. In particular, both examples use the same OPE potential for the long-range interaction. This suggests that the claims in the literature that pions cannot be treated perturbatively [22] are at least oversimplified, as the perturbative expansion works well in the two-Yukawa example.

It is clear that the three-Yukawa example fails for several reasons. First of all, $p \widetilde{r}_{0}$ is assumed small at $p \sim m_{\pi}$, whereas numerically it is large. Therefore, the fact that $p \widetilde{r}_{0}$ is not kept to all orders in the KSW expansion is a practical problem. A possible resolution is to adopt the method for summing the effects of large $r_{0}$ by means of a dibaryon field, as discussed in Refs. [23,8]. Secondly, the effective range expansion plot in Fig. (1 shows that, 
even if the effects of a large $\widetilde{r}_{0}$ are summed to all orders, the EFT to NNLO may still not give accurate predictions for the phase shift above $p \sim m_{\pi}$. This depends on the particular form of the short-distance physics and has nothing to do with the pion. Finally, the very nature of the three-Yukawa potential implies that even if one sums the effects of $\widetilde{r}_{0}$ to all orders and pushes beyond NNLO to include $\widetilde{v}_{2}$, the convergence will never extend to $p \sim m_{\rho}$, but instead will fail at $p \sim m_{\sigma} / 2=250 \mathrm{MeV}$. This obstacle does not depend on how one chooses to perform the EFT expansion, and can only be surmounted by explicitly including the $\sigma$ field in the EFT. If chiral symmetry cannot describe the $\sigma$ as irreducible two-pion effects, an EFT including explicit $\sigma$ mesons would have little to offer beyond conventional modeling techniques for nuclear physics.

We have not included an analysis of the real data in this section, since to date the full amplitude at NNLO has not been calculated. In particular, the contributions from radiation pions (on-shell, propagating pions) [24, has not been computed to this order. When these terms are known, then our algorithm can be readily applied to the full NNLO amplitude, testing whether the data exhibits the same behavior as the three-Yukawa model. Much of the difficulties encountered in our three-Yukawa model would be alleviated if radiation pions, which are absent in the potential models, are found to make a significant contribution to $r_{0}$; however, we have no reason to expect this to happen.

\section{DISCUSSION}

In this paper, we have examined the nature of the KSW expansion in a systematic fashion by using models for potential scattering between "nucleons". These model potentials contain both short- and long-range interactions and by construction give rise to a large scattering length, which can lead to complications in the implementation of power counting methods. By beginning with an explicitly solvable toy model, we motivated an algorithm for fixing the unknown coupling constants of the EFT, directly relating them to the effective range expansion for scattering from the short-distance potential alone. This procedure is similar to the modified effective range expansion [13,14]. We then applied this algorithm to three different models.

We find that for a realistic pion mass and coupling, the quality of the expansion is good when the relation $m_{\pi} \widetilde{r}_{0}<1$ is valid, where $\widetilde{r}_{0}$ is the effective range due to the short-range part of the potential alone. An exception is seen at low momentum, where the strict perturbative pion expansion fails due to the delicate interplay between short-range and pion interactions that gives rise to a large scattering length. This problem is dealt with by reordering the expansion to correctly account for the scattering length or pole in the amplitude at LO, as discussed previously in Refs. [15, 16]. The algorithm we provide for fixing the contact interactions of the EFT correctly account for this reordering.

It remains to be seen, however, if $\widetilde{r}_{0}$ needs to be large for real ${ }^{1} S_{0} N N$ scattering, or whether the large effective range $r_{0}$ gets sizable contributions from radiation pion effects. In potential models, however, $\widetilde{r}_{0}$ does have to be large and results in the KSW expansion doing poorly, a discovery made previously in Ref. [20]. In this case, the Weinberg expansion, which sums $\widehat{C}_{2}$ (and hence $\widetilde{r}_{0}$ ) to all orders, may do better. However, as the Weinberg procedure involves summing the extended pion interaction to all orders at LO in the EFT expansion, it seems to offer only modest benefits over conventional potential model techniques for 
systems with more than two nucleons. In order to sum $\widetilde{r}_{0}$ to all orders while still treating pions perturbatively, it may be advantageous to make use of the dibaryon field as discussed in [23,8], and used to advantage in the three-body problem [25,26]. A revised power counting scheme, perhaps treating $\widetilde{r}_{0} \sim 1 / m_{\pi}$, remains to be worked out.

\section{Acknowledgements}

D.K. would like to thank T. Mehen, G. Rupak, N. Shoresh, I. Stewart, and M. Wise for useful discussions, and is supported in part by the U.S. Dept. of Energy under Grants No. DOE-ER-40561 and No. DE-FG03-92ER40689; he thanks the Santa Cruz Institute for Particle Physics and the TH Division of CERN for their hospitality during various stages of this work. J.S. would like to thank R. Furnstahl for useful discussions and is supported by the National Science Foundation under Grants No. PHY-9511923 and PHY-9258270.

\section{APPENDIX A: SUMMING UP TOY PION GRAPHS}

The toy pions of Eq. (10) have a simple enough form to allow an analytic summation of all contributions. Following the discussion in Ref. [12], there are three classes of graphs to compute: i) pion ladders, ii) a single vertex of the short-distance potential dressed by pion exchange on one side, and iii) two vertices of the short-distance potential with pion exchange in between.

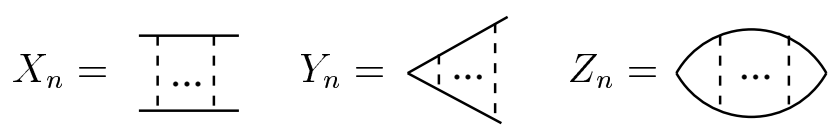

They take the form

$$
\begin{aligned}
& X_{n} \equiv\left\langle\mathbf{p}\left|\hat{V}_{L}\left(\hat{G}_{E} \hat{V}_{L}\right)^{n}\right| \mathbf{p}^{\prime}\right\rangle=-\frac{4 \pi}{M m_{\pi}}\left(\frac{g_{\pi} \sin ^{2} \xi}{\xi^{2}}\right)\left(\frac{g_{\pi} e^{i \xi} \sin \xi}{\xi}\right)^{n} \\
& Y_{n} \equiv\left\langle\mathbf{r}=0\left|\left(\hat{G}_{E} \hat{V}_{L}\right)^{n}\right| \mathbf{p}^{\prime}\right\rangle=\left(\frac{g_{\pi} e^{i \xi} \sin \xi}{\xi}\right)^{n}, \\
& Z_{n} \equiv\left\langle\mathbf{r}=0\left|\hat{G}_{E} \hat{V}_{L} \hat{G}_{E}\left(\hat{V}_{L} \hat{G}_{E}\right)^{n}\right| \mathbf{r}^{\prime}=0\right\rangle=-\frac{M m_{\pi}}{4 \pi}\left(g_{\pi} e^{2 i \xi}\right)\left(\frac{g_{\pi} e^{i \xi} \sin \xi}{\xi}\right)^{n}
\end{aligned}
$$

where $p^{2}=\left(p^{\prime}\right)^{2}=M E, \xi=p / m_{\pi}$, and we have projected onto the $s$-wave.

These graphs are trivially summed as they form a geometric series. Using the PDS subtraction scheme, the final result for the exact Feynman amplitude in the effective theory with pions is

$$
\mathcal{A}^{\mathrm{eff}}=-\sum_{n=0}^{\infty} X_{n}-\frac{\frac{4 \pi}{M} \widehat{C}\left(p^{2} ; \mu\right)\left[\sum_{n=0}^{\infty} Y_{n}\right]^{2}}{1-\widehat{C}\left(p^{2} ; \mu\right)\left[-\mu-i p+\frac{4 \pi}{M} \sum_{n=0}^{\infty} Z_{n}\right]}
$$




$$
=\mathcal{A}_{L}+\frac{4 \pi / M}{f(\xi)^{2}\left[-\mu-i p-\widehat{C}^{-1}\left(p^{2} ; \mu\right)\right]-g_{\pi} m_{\pi} e^{2 i \xi} f(\xi)},
$$

where we have defined $f(\xi)$ and $\mathcal{A}_{L}$ as in Eqs. (16, 17).

\section{APPENDIX B: TOY PION AMPLITUDE TO NNLO}

Taking the exact EFT result for the toy pions Eq. (22) and expanding in powers of $Q$ according to the rules of $\S \amalg \mathrm{IC}$ and $\S \amalg \mathrm{IIC} 3$, the first few terms are

$$
\begin{gathered}
\mathcal{A}_{-1}=-\frac{\frac{4 \pi}{M} \widehat{C}_{0}^{[-1]}}{1+\widehat{C}_{0}^{[-1]}(\mu+i p)}, \\
\mathcal{A}_{0}=\left(\widehat{C}_{2}^{[-2]} p^{2}+\widehat{C}_{0}^{[0]}\right) \frac{d \mathcal{A}_{-1}}{d \widehat{C}_{0}^{[-1]}-X_{0}+2 \mathcal{A}_{-1} Y_{1}-\mathcal{A}_{-1}^{2} Z_{0},} \\
\mathcal{A}_{1}=\left(\widehat{C}_{4}^{[-3]} p^{4}+\widehat{C}_{2}^{[-1]} p^{2}+\widehat{C}_{0}^{[1]}\right) \frac{d \mathcal{A}_{-1}}{d \widehat{C}_{0}^{[-1]}}+\frac{1}{2}\left(\widehat{C}_{2}^{[-2]} p^{2}+\widehat{C}_{0}^{[0]}\right)^{2} \frac{d^{2} \mathcal{A}_{-1}}{d \widehat{C}_{0}^{[-1]}} \\
+\left(\widehat{C}_{2}^{[-2]} p^{2}+\widehat{C}_{0}^{[0]}\right) \frac{d}{d \widehat{C}_{0}^{[-1]}}\left(2 \mathcal{A}_{-1} Y_{1}-\mathcal{A}_{-1}^{2} Z_{0}\right) \\
-X_{1}+\mathcal{A}_{-1}\left(Y_{1}^{2}+2 Y_{2}\right)-\mathcal{A}_{-1}^{2}\left(2 Y_{1} Z_{0}+Z_{1}\right)+\mathcal{A}_{-1}^{3} Z_{0}^{2} .
\end{gathered}
$$

Using the values for the $X_{n}, Y_{n}$, and $Z_{n}$ in Appendix $\mathbb{A}$, these expressions can be rewritten as

$$
\begin{aligned}
(p \cot \delta)_{\mathrm{LO}}= & -\frac{1}{a} \\
(p \cot \delta)_{\mathrm{NLO}}= & (p \cot \delta)_{\mathrm{LO}}+\frac{\left(m_{\pi}-1 / a\right)^{2}}{\Lambda_{\pi}}+\frac{2 p^{2}}{3 m_{\rho}}-\frac{m_{\pi}^{2}}{\Lambda_{\pi}}\left(\cos \xi-\frac{\sin \xi}{p a}\right)^{2}, \\
(p \cot \delta)_{\mathrm{NNLO}}= & (p \cot \delta)_{\mathrm{NLO}}+\frac{\left(m_{\pi}-1 / a\right)^{3}}{\Lambda_{\pi}^{2}}-\frac{p^{2}}{3 a m_{\rho}^{2}}+\frac{m_{\pi}^{3}}{\Lambda_{\pi}^{2}} \frac{\sin \xi}{\xi}\left(\cos \xi-\frac{\sin \xi}{p a}\right)^{3} \\
& -\frac{2 m_{\pi}}{\Lambda_{\pi}} \frac{\sin \xi}{\xi}\left(\frac{\left(m_{\pi}-1 / a\right)^{2}}{\Lambda_{\pi}}+\frac{2 p^{2}}{3 m_{\rho}}\right)\left(\cos \xi-\frac{\sin \xi}{p a}\right) .
\end{aligned}
$$




\section{REFERENCES}

[1] Nuclear Physics with Effective Field Theory, ed. R. Seki, U. van Kolck, and M. J. Savage (World Scientific, 1998);

U. van Kolck, nucl-th/9902015, and references therein.

[2] S. Weinberg, Phys. Lett. B 251, 288 (1990); Nucl. Phys. B 363, 3 (1991); Phys. Lett. B 295, 114 (1992).

[3] C. Ordonez and U. van Kolck, Phys. Lett. B 291, 459 (1992);

C. Ordonez, L. Ray, and U. van Kolck, Phys. Rev. Lett. 72, 1982 (1994); Phys. Rev. C 53, 2086 (1996);

U. van Kolck, Phys. Rev. C 49, 2932 (1994).

[4] T. S. Park, D. P. Min, and M. Rho, Phys. Rev. Lett. 74, 4153 (1995); Nucl. Phys. A 596, 515 (1996).

[5] J. V. Steele and R. J. Furnstahl, Nucl. Phys. A 637, 16 (1998).

[6] D. B. Kaplan, nucl-th/9804061.

[7] M. C. Birse, J. A. McGovern, and K. G. Richardson, hep-ph/9807302.

[8] D. B. Kaplan, M. J. Savage, and M. B. Wise, Phys. Lett. B 424, 390 (1998); Nucl. Phys. B 534, 329 (1998); D. B. Kaplan, lecture delivered at Baryons '98, Bonn, Sept. 22, 1998, nucl-th/9901003.

[9] T. D. Cohen, Phys. Rev. C 55, 67 (1997);

D. R. Phillips and T. D. Cohen, Phys. Lett. B 390, 7 (1997);

S. R. Beane, T. D. Cohen, and D. R. Phillips, Nucl. Phys. A 632, 445 (1998); Ann. Phys. 263, 255 (1998);

K. A. Scaldeferri, D. R. Phillips, C. W. Kao, and T. D. Cohen, Phys. Rev. C 56, 679 (1997).

[10] H. A. Bethe, Phys. Rev. 76, 38 (1949).

[11] J.-W. Chen, G. Rupak, and M. J. Savage, nucl-th/9902056.

[12] D. B. Kaplan, M. J. Savage, and M. B. Wise, Nucl. Phys. B 478, 629 (1996).

[13] H. van Haeringen and L. P. Kok, Phys. Rev. A 26, 1218 (1982).

[14] J. V. Steele and R. J. Furnstahl, Nucl. Phys. A 645, 439 (1999).

[15] T. Mehen and I. W. Stewart, Phys. Lett. B 445, 378 (1999).

[16] G. Rupak and N. Shoresh, nucl-th/9902077.

[17] D. B. Kaplan, M. J. Savage and M. B. Wise, Phys. Rev. C 59, 617 (1999).

[18] T. Mehen and I. W. Stewart, private communication.

[19] J. V. Steele and R. J. Furnstahl, in preparation.

[20] T. D. Cohen and J. M. Hansen, Phys. Lett. B 440, 233 (1998); Phys. Rev. C 59, 13 (1999); nucl-th/9901065.

[21] T. D. Cohen, nucl-th/9904052.

[22] J. Gegelia, nucl-th/9802038; hep-th/9804189; nucl-th/9805008; nucl-th/9806028.

[23] D. B. Kaplan, Nucl. Phys. B 494, 471 (1997).

[24] T. Mehen and I. W. Stewart, nucl-th/9901064.

[25] P. F. Bedaque and U. van Kolck, Phys. Lett. B 428, 221 (1998);

P. F. Bedaque, H.-W. Hammer, and U. van Kolck, Phys. Rev. C 58, 641 (1998); Nucl. Phys. A 646, 444 (1999); Phys. Rev. Lett. 82, 463 (1999).

[26] J. Gegelia, nucl-th/9903030. 